Update on specifications for NIF ignition targets and their rollup into an error budget

Steven W. Haan, Mark C. Herrmann, Peter A. Amendt, Debra A. Callahan, Thomas R. Dittrich, M. John Edwards, Ogden S. Jones, M.Marty Marinak, David H. Munro, Stephen M. Pollaine, Jay D. Salmonson, Brian K. Spears, Laurance J. Suter

July 12,2005

Sixteenth Target Fabrication Specialists Meeting Scottsdale, AZ, United States May 1, 2005 through May 5, 2005 
This document was prepared as an account of work sponsored by an agency of the United States Government. Neither the United States Government nor the University of California nor any of their employees, makes any warranty, express or implied, or assumes any legal liability or responsibility for the accuracy, completeness, or usefulness of any information, apparatus, product, or process disclosed, or represents that its use would not infringe privately owned rights. Reference herein to any specific commercial product, process, or service by trade name, trademark, manufacturer, or otherwise, does not necessarily constitute or imply its endorsement, recommendation, or favoring by the United States Government or the University of California. The views and opinions of authors expressed herein do not necessarily state or reflect those of the United States Government or the University of California, and shall not be used for advertising or product endorsement purposes. 


\title{
Update on specifications for NIF ignition targets, and their rollup into an error budget
}

\author{
S. W. Haan, M. C. Herrmann, P. A. Amendt, D. A. Callahan, T. R. Dittrich, M. J. Edwards, O.S. Jones, M. \\ M. Marinak, D. H. Munro, S. M. Pollaine, J. D. Salmonson, B. K. Spears, and L. J. Suter
}

Lawrence Livermore National Laboratory, Livermore CA

\begin{abstract}
Targets intended to produce ignition on NIF are being simulated and the simulations are used to set specifications for target fabrication. Recent design work has focused on designs that assume only 1.0 MJ of laser energy instead of the previous 1.6 MJ. To perform with less laser energy, the hohlraum has been redesigned to be more efficient than previously, and the capsules are slightly smaller. The main-line hohlraum design now has $\mathrm{a} \mathrm{SiO}_{2}$ foam fill, a wall of U-Dy$\mathrm{Au}$, and shields mounted between the capsule and the laser entrance holes. Two capsule designs are being considered. One has a graded doped $\mathrm{Be}(\mathrm{Cu})$ ablator, and the other graded doped $\mathrm{CH}(\mathrm{Ge})$. Both can perform acceptably with recently demonstrated ice layer quality, and with recently demonstrated outer surface roughness. Smoothness of the internal interfaces may be an issue for the $\mathrm{Be}(\mathrm{Cu})$ design, and it may be necessary either to polish partially coated shells or to improve process control so that the internal layers are smoother. Complete tables of specifications are being prepared for both targets, to be completed this fiscal year. All the specifications are being rolled together into an error budget indicating adequate margin for ignition with the new designs.
\end{abstract}

\section{INTRODUCTION}

This presentation is an update on the fabrication specifications as they result from the indirect drive ignition target design effort at LLNL. ${ }^{12}$ Recent work has been in response to three changes in the plan for achieving ignition: (i) We now plan to use only 1.0 MJ of laser energy, instead of the previously assumed 1.6 MJ. This is in order to attempt ignition within the first year after the facility is up, and since the laser will be relatively new we should not assume that we can operate it at full power and energy. (ii) In order to save on money and complexity, we are going to assume that the capsules do not require cryogenic transport after fill. In particular, fill tubes are now part of the baseline. (iii) For several reasons (fielding simplicity, laser-plasma interactions, and convection) the mainline target is now filled with $\mathrm{SiO} 2$ foam rather than helium.

New targets reflecting these changes have been designed, and a complete rollup of specifications on them will be done by the end of FY05. Preliminary specifications are described below.

The current point design is shown in Fig. 1. It differs from previous designs in having the foam fill, as described above, and having shields between the capsule and the laser entrance holes. These improve the efficiency of the hohlraum and reduce intrinsic P2 asymmetry. How they affect the overall symmetry tune is still work in progress. The hohlraum is assumed to be made of a "cocktail" mixture of U, Au, and Dy. These two changes to the hohlraum design improve the efficiency enough that the capsule is only $5 \%$ smaller than the previous baseline, in radius, instead of being $15 \%$ smaller as it would be by straight scaling from 1.6 MJ.

In addition to this point design, we have done simulations of four other hohlraums and
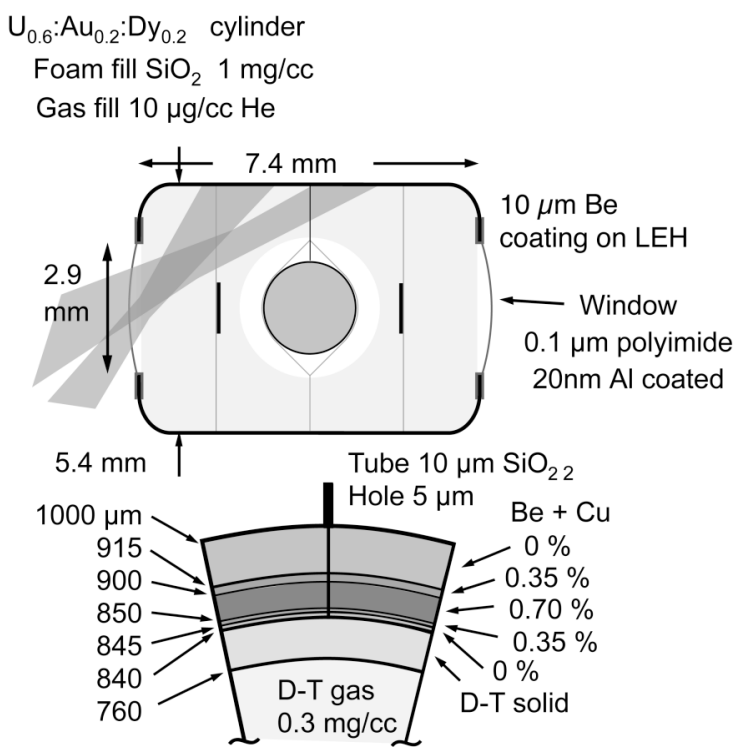

Figure 1. New point design target that uses $1.0 \mathrm{MJ}$ of laser energy 
will be considering a number of variants in the future. Possibilities are: with and without LEH shields; a liner on the hohlraum instead of the foam fill - the liner could be higher density foam, of full density glass or beryllium; and traditional gas fill instead of foam or a liner. The cocktail wall material is still being optimized, and will almost certainly be different than the current preliminary optimization. Likely possibilities are removing the Dy, and replacing the current mixture with some combination of layers of $\mathrm{Au}$ and $\mathrm{U}$. Currently no $\mathrm{Nb}$ is assumed in the $\mathrm{U}$, but that is likely to change as well.

In addition to these options on the hohlraum, we plan to maintain several options on the capsule specifics. Possibilities here are: uniformly doped $\mathrm{Be}(\mathrm{Cu})$; graded doped $\mathrm{CH}(\mathrm{Ge})$; diamond, uniformly or graded doped with a high- $\mathrm{Z}$ material TBD. The $\mathrm{CH}$ and diamond capsules, as currently designed, are shown in Fig. 2.

Our goal is to have a complete set of specifications for the target shown in Fig. 1 by the end of FY05. For the other targets, as shown in Fig. 2, and for the other hohlraum options, we plan to have preliminary specifications documented at that time.

The remainder of this paper describes how specification are being developed, and the preliminary specifications for the $\mathrm{Be}($ graded $\mathrm{Cu})$ and $\mathrm{CH}$ (graded $\mathrm{Ge})$ targets.
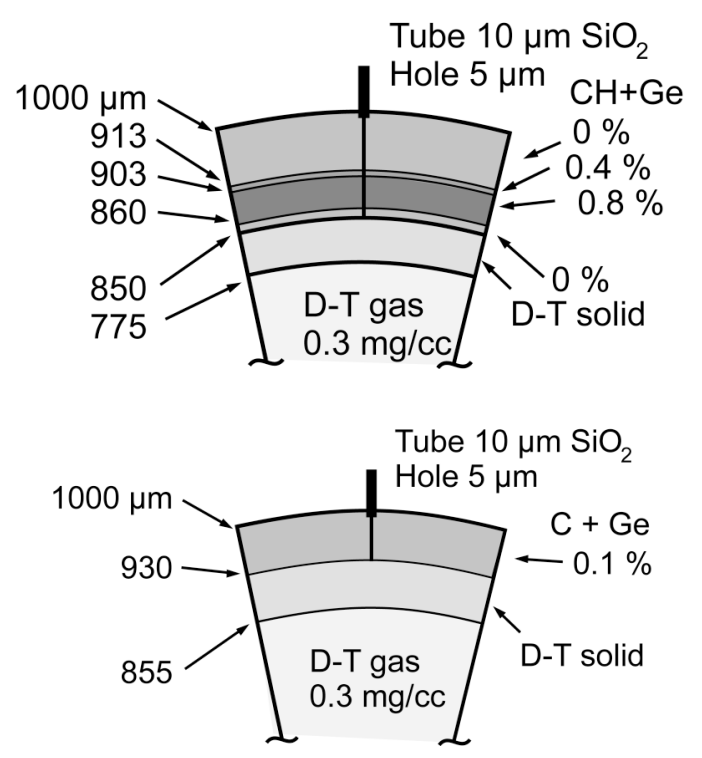

Figure 2. Alternate designs using Ge-doped $\mathrm{CH}$ and $\mathrm{C}$ ablator. The $\mathrm{C}$ design assumes diamond-like density for the ablator

\section{PROCEDURE FOR SETTING SPECS}

Specifications are being set using simulations of the growth and impact of the various deviations between plausible expected reality and the ideal implosion. Three kinds of simulations are being used.

\section{(i) One dimensional simulations}

In one dimension (1D) we consider variations in dimensions, pulse shape, densities, dopant concentrations, and drive spectrum. These are considered one at a time, and in random combinations assuming Gaussian statistics with specified root mean square deviations. For past designs, the 1D variations have been considered in combination with $2 \mathrm{D}$ errors; this will be done in the future for the new design, eventually in 3D.

\section{(ii) Growth factors and linear analysis}

In 2D, simulations are done with infinitesimal perturbations to determine "growth factors," i.e. the final perturbations that result, being proportional to the initial perturbation, with the proportionality factor being the growth factor. Perturbations are imposed in the form of single Legendre polynomial modes; the growth factor for any 3D spherical harmonic perturbation is thereby known. Growth factor curves vs. mode number are determined for initial perturbations on each of the seven interfaces, and for density variations. The density variations are used to set specifications for voids in the beryllium and DT. For these we must assume a radial dependence; so far we have considered density perturbations independent of radius in the ice, in the beryllium shell overall, and in the first layer of the beryllium. The perturbations grow to be very similar to those seeded by surface perturbations at the nearest surface, with the same initial column density variation.

The growth factors are combined with assumed spectra of initial perturbations, to determine final perturbations. This linear analysis is fully $3 \mathrm{D}$, and is very accurate if the perturbations are small enough to remain linear (it is less accurate for the density variations, since they can only be simulated for an assumed radial variation which does not correspond to voids in the same "exact" way that surface perturbations are described in the simulations). Final perturbations are considered at two times: peak velocity and ignition time. At ignition time the perturbations are only very slightly nonlinear and the linear analysis provides an excellent 

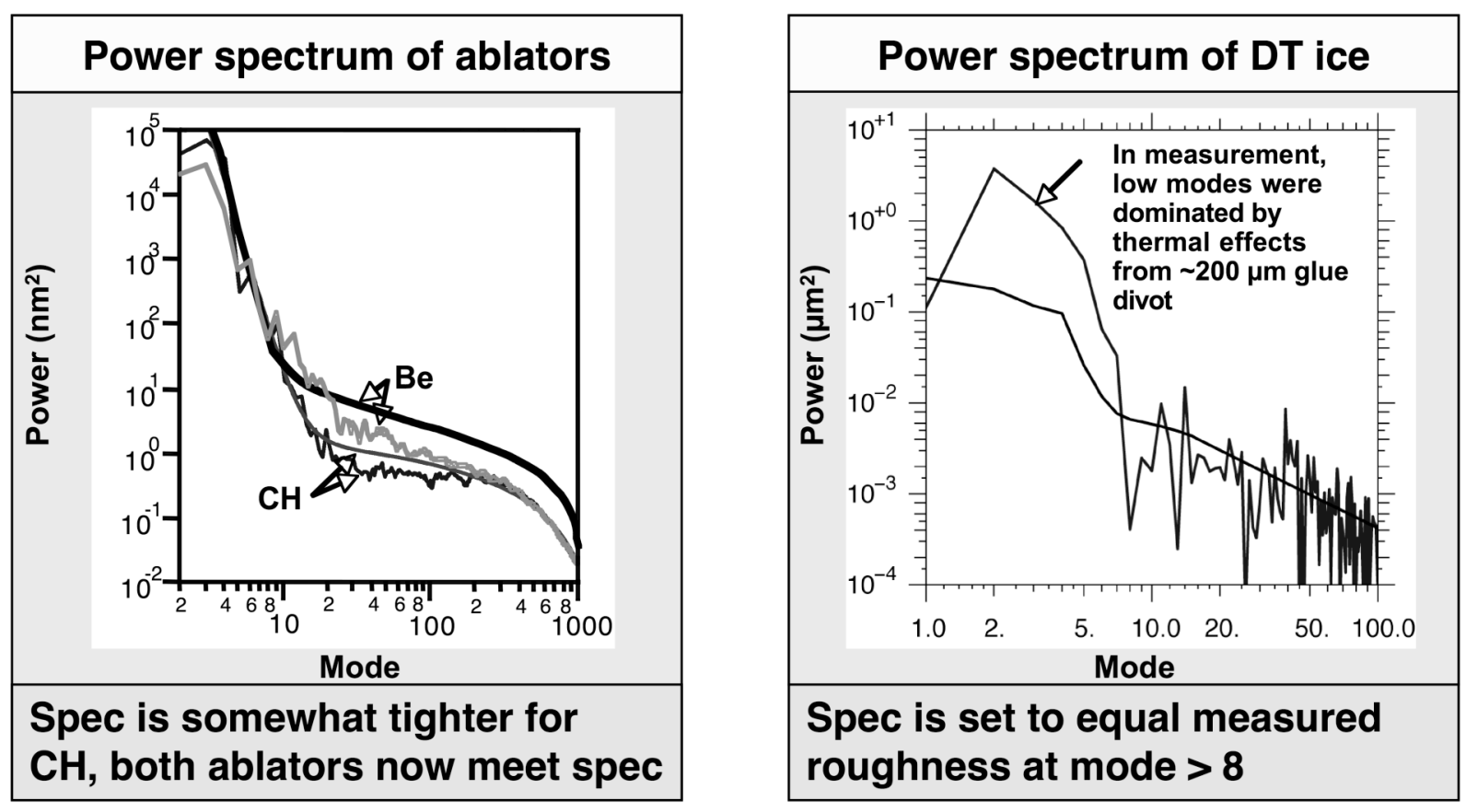

Fig. 3. Initial surface specifications for the outer surface roughness, and for the ice, for the $\mathrm{Be}(\mathrm{graded} \mathrm{Cu})$ and $\mathrm{CH}$ (graded $\mathrm{Ge})$ targets

estimate of the final perturbations. They are predominantly in modes below about 20; which perturbations dominate will be described below when the specifications are described.

At peak velocity the perturbations are at much shorter wavelength (modes 100-400). Multi-mode simulations, with realistic initial perturbations (see the next section) indicate that growth is actually linear up to modes about 500 . (The perturbations are large compared to the wavelength, but the growth is largely due to a "taffy pull" expansion that does not go into nonlinear saturation in the way that RayleighTaylor perturbations are known to in incompressible fluids.) Linear analysis also predicts substantial amplitude at even higher modes. These perturbations grow on the interface between the fuel and the beryllium, at the late stages of the acceleration when that interface is unstable. Their growth varies substantially from one target design to another. ${ }^{3}$ At very high modes (above 1000) the growth is expected to be reduced by diffusion and viscosity. At intermediate modes - 500 to 1000 - the best approximation available to us seems to be to assume nonlinear saturation. ${ }^{4}$ That was done for the numbers reported below. The targets are being redesigned to reduce the sensitivity to this are of uncertainty.

(iii) Large amplitude 2D simulations
The linear analysis procedure tells us how large the perturbations are expected to be, but does not tell us how large they are allowed to be. For that we use simulations with larger-thanspecified initial perturbations, in order to find the "cliff" in performance. In the past, for other designs, these simulations have been done in $3 \mathrm{D}$, and $3 \mathrm{D}$ simulations will be done for the new designs in coming months. Now they have been done in $2 \mathrm{D}$. The character of the final perturbations is only very weakly dependent on how the perturbations were seeded, since the growing perturbations settle into an eigenmode, and "find" the dominant modes regardless of seed. The main parameters determining the performance are the overall amplitude of the perturbations, and the dominant mode, regardless of seed except insofar as the combination of amplitudes of initial seeds determines those two final characteristics.

With these large-amplitude simulations we see the same two failure modes described above. The perturbations at peak velocity amount to beryllium "bubbles" penetrating the fuel from the outside. The fuel that is thereby contaminated does not burn, later on after ignition. Thus this penetration must be kept small enough to allow the needed yield. This is not necessarily hard failure, and in principle it could end up that we accept considerable mix penetration and yield reduction. Dominant modes are several hundred. 
Currently specifications are set so that the yield reduction is less than about $10 \%$. We are working to redesign the targets to reduce the high mode growth. The other failure mode is at ignition time, when spikes of cold high density DT penetrate the hot spot and cool it as it is trying to ignite. This is hard failure as it can prevent ignition. Dominant modes are 5-25. This failure mode is coupled with 1D errors, since 1D errors reduce margin, delay ignition, and increase the deceleration Rayleigh-Taylor growth. In simulations that combine a $1 \mathrm{D}$ error with a $2 \mathrm{D}$ error, we find that their impact is close to being described via a quadrature sum of the two effects. In the specifications as described below, the 1D and 3D errors are almost equally balanced, with net impact being about $\sqrt{2}$ times each individual contributor.

\section{SPECIFICATIONS FOR 1.0 MJ TARGETS}

The procedure just described has been applied to the $\mathrm{Be}$ (graded $\mathrm{Cu}$ ) and $\mathrm{CH}$ (graded $\mathrm{Ge}$ ) targets shown above. Specifications that result in acceptable performance are similar to what has been demonstrated for these materials. The ablator roughness specifications are shown in Fig. 3. The Be target is allowed to be somewhat rougher than $\mathrm{CH}$, because the implosion is more stable. The noisy curves overplotted are characterization results for the two materials (the beryllium has been polished). ${ }^{5}$ Except for a possible issue with Be at modes around 15, the demonstrated surfaces meet the specifications. The ice roughness requirement is in Fig. 4. In this case there is no reason to expect that the ice can be smoother in $\mathrm{CH}$ than in Be. The noisy curve in this case is the demonstrated ice roughness. At low modes the spec is tighter than has been demonstrated, but the experiments so far have been dominated by a large fill tube that affects the thermal distribution.

There are similar curves for the internal interfaces in the graded-doped beryllium, shown in Fig. 4.

For voids in the beryllium, the current specifications assume that the voids are randomly located, that they have a characteristic volume, and that the density is known. This then determines the number of voids, and simple statistics describes the variations in column density. For the beryllium we specify that the void fraction is less than $1 \%$, and that the characteristic void volume is less than $0.3 \mu \mathrm{m}^{3}$.
Actually only the product, (void density) * (void volume) determines the column density variations. We expect the voids to be characterized by radiographic transmission, and if our assumptions about random location etc. are not valid, then to first order their impact on the implosion is still adequately characterized: the impact on the implosion, and the signature in radiographic characterization are both determined by the column density. So the specification is "the voids need have the same impact on integrated column density as if they were randomly located with volume $0.3 \mu \mathrm{m}^{3}$ and void fraction $1 \%$." We have estimated the variations in optical depth that arise in radiographic characterization of the shells, and the current plan of measuring to $1: 10^{4}$ should be adequate to see the currently specified voids. To a very rough first approximation the same is true for opacity variations, although in this case the relative impact is somewhat different. In the case of opacity variations, it appears that radiographic characterization is actually more sensitive than necessary, so that variations in oxygen (for example) will be constrained not by implosion requirements directly, but by the need to be able

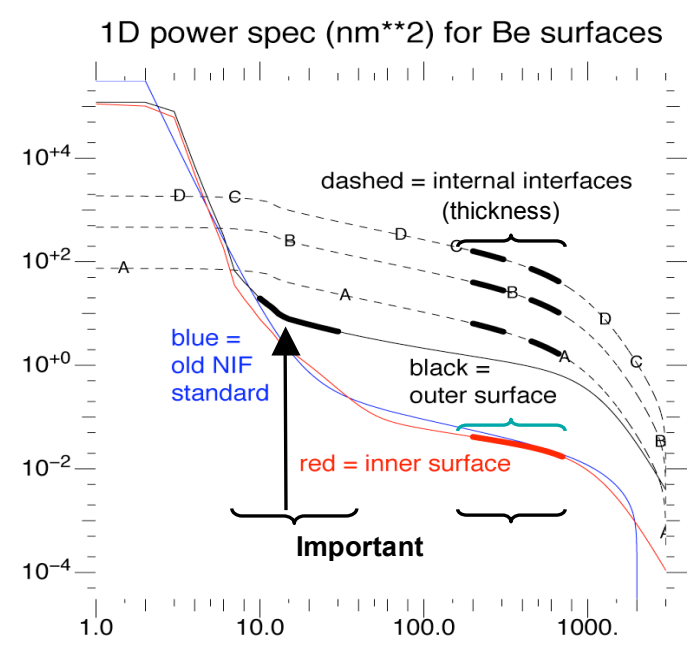

Figure 4. Power spectra for beryllium surfaces. Specifications on internal surfaces are on the layer thickness.

to characterize void structure in the beryllium. It has been suggested that the Be void fraction could be as large as 5\%. In that case the targets will need to be redesigned. With such a large void fraction the voids would have to be very fine, approximately $0.06 \mu \mathrm{m}^{3}$ as a 
characteristic volume. We do not know how much the overall performance will be affected by a redesign with a lower nominal density.

Simulations have continued of fill tubes and holes. The currently assumed configuration of a 10-micron glass tube, over a 5-micron hole in the ablator, appears to be acceptable for both ablator materials.

\section{SUMMARY}

Ignition targets have been redesigned to use less laser energy, foam-filled hohlraums, and fill tubes. Specifications are being set for the new targets and appear reasonable. Fully documented detailed specifications will be available at the end of this fiscal year.

\section{ACKNOWLEDGEMENT}

This work was performed under the auspices of the U.S. Department of Energy by the University of California, Lawrence Livermore National Laboratory under contract No. W-7405-Eng-48.

\section{REFERENCES}

${ }^{1}$ J. Lindl, Inertial Confinement Fusion, SpringerVerlag, New York (1998).

2 John D. Lindl, Peter Amendt, Richard L.

Berger, S. Gail Glendinning, Siegfried H.

Glenzer, Steven W. Haan, Robert L. Kauffman, Otto L. Landen, and Laurence J. Suter, "The physics basis for ignition using indirect-drive targets on the National Ignition Facility," Phys. Plasmas 11, 339 (2004).

${ }^{3}$ S. W. Haan, M. C. Herrmann, T. R. Dittrich, A. J. Fetterman, M. M. Marinak, D. H. Munro, S. M. Pollaine, J. D. Salmonson, G. L. Strobel, and L. J. Suter, "Increasing robustness of indirect drive capsule designs against short wavelength hydrodynamic instabilities," Phys. Plasmas 12, 056316 (2005).

${ }^{4}$ S. W. Haan, "Onset of nonlinear saturation for Rayleigh-Taylor growth in the presence of a full spectrum of modes," Phys. Rev. A 39, 5812 (1989).

${ }^{5}$ A. Nikroo and R. Cook, private communication(2005). 